\title{
Aprendizaje y Evaluación Basada en Proyectos en una Asignatura de Dirección de Operaciones
}

\author{
Sofía Estelles-Miguel ${ }^{\mathrm{a}}$, José Miguel Albarracín Guillem ${ }^{\mathrm{b}}$, Marta Palmer Gato ${ }^{\mathrm{c}}$, Marta \\ Peris-Ortiz $^{\mathrm{d}}$ \\ Departamento de Organización de Empresas de la Universitat Politècnica de València

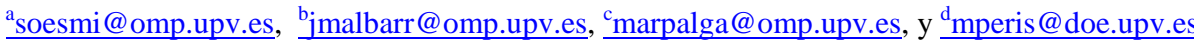

\begin{abstract}
In the actual context of Economics and Business degrees, the subject Operation Management provide a wide variety of tools which appear to be narrowly related to several main competences such as creativity, problem solving, decision-making and information management. In this way the authors of this paper pretending that the students learning Operations Management by doing Operation Management.

In order to do this, the authors have designed a project based learning and the student by teamworks will have to develop during the subject. Team projects can play a main role in the teaching-learning process, since they allow students cooperating in the development of the real project of Operations Management, proving their ability to work in team, using creativity and others tools as suggested by employers. In the present paper we summarize our experiences during the course 2014/2015 in the subject Operations and Production Management at the Faculty of Business Administration in the Universitat Politècnica de València.
\end{abstract}

Keywords:

Operation Management, project-based learning, teaching-learning process, competences, skills.

\section{Resumen}

En el contexto actual de los grados de Economía y Empresa, la asignatura Dirección de Operaciones proporciona una amplia variedad de herramientas estrechamente relacionadas con varias competencias como son creatividad, resolución de problemas, toma de decisiones y gestión de la información. En este contexto los autores de este trabajo pretenden que los estudiantes aprendan Dirección de Operaciones haciendo Dirección de Operaciones.

Para ello, los autores han diseñado un aprendizaje basado en proyectos que los estudiantes por equipos de trabajo desarrollaran durante la asignatura. Los projectos por equipos pueden hacer un papel muy importante en el proceso de enseñanza-aprendizaje, ya que permiten a los estudiantes cooperar en el desarrollo de un proyecto real de Dirección de Operaciones, probando su habilidad de trabajar en equipo, usando la creatividad y otras herramientas que han sido sugeridas por los empleadores. En el presente 
artículo resumimos la experiencia realizada en el curso 2014/2015 en la asignatura Dirección de Producción y Operaciones en la Facultad de Administración y Dirección de Empresas de la Universitat Politècnica de València.

Palabras clave: Dirección de Operaciones, Aprendizaje Basado en Proyectos, Proceso de ensenza-aprendizaje, competencias, habilidades

\section{Introducción}

Aprovechando el proceso de adaptación al nuevo Espacio Europeo de Educación Superior (EEES) y el cambio desde las Licenciaturas a los Grados en la Facultad de Administración y Dirección de Empresas (FADE) algunas asignaturas cambiaron sus nombres, su cantidad de docencia, el curso en el que se impartían y otros. La asignatura de Dirección de Producción y Logística (DPL), asignatura que se impartía en $4^{\circ}$ curso de la Licenciatura se transformo en la asignatura de Dirección de Producción y Operaciones (DPO) que se imparte en $2^{\circ}$ curso de Grado, además la asignatura se vio reducida de 9 a 6 ECTS, ambas asignaturas tanto la vieja como la nueva son troncales, es por todo ello por lo que los profesores decidieron reestructurar no sólo el nombre y el curso en el que se impartía sino más cosas para adaptarla a los nuevos alumnos de la misma. La adaptación al EEES implica que las asignaturas sean diseñadas en competencias y en objetivos de aprendizaje, esto afecta no solo a la metodología de enseñanza-aprendizaje sino también a la evaluación de la misma. Es por ello, que los profesores de esta asignatura replantearon una parte de la misma revisando no sólo la forma en la que era impartida, sino también la forma de evaluarla.

Otro cambio importante introducido por la EEES es centrar la atención no solo en el aprendizaje de la asignatura, sino también en el desarrollo de competencias y habilidades que van a preparar a los estudiantes en su integración en el mundo laboral. Pero la gestión de las competencias genera numerosas dificultades (Levy-Leboyer y Prieto, 1997 y Estelles-Miguel et al., 2013), y es su consecución lo que puede y debe estimular a los profesores, para la aplicación de estas nuevas metodologías ayudandose de las TIC (Garai et al, 2003 y Hernández Pina et al., 2005). Se necesita cambiar la dinámica de las clases basadas en el aprendizaje memorístico de conocimientos, a otra en la que los estudiantes sean una parte activa pasando a ser protagonistas y responsables de su propio aprendizaje en lugar de sujetos pasivos (Zabala y Arnau, 2007).

Las asignaturas relacionadas con el ámbito de la empresa pueden explicarse utilizando metodologías muy variadas. Aunque la forma clásica ha sido la de exposiciones magistrales debido a la masificación de las aulas (Barbera et al, 2015). En el presente trabajo se explica el diseño, la aplicación y los resultados de un cambio de modelo en la parte más teórica de la asignatura, con lo que se ha conseguido que esta parte que los alumnos veían como más difícil de entender ya que no tenían un conocimiento de las empresas, se ha trasladado a aprendizaje basado en proyectos de forma que los alumnos aprendan haciendo y de esta forma la asignatura sea más facilmente comprensible por parte de los alumnos y más práctica y tangible.

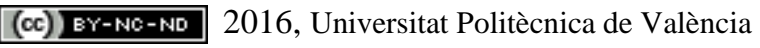


La asignatura en la que se ha desarrollado esta innovación educativa es una asignatura de carácter obligatorio que se compone de 3 créditos de teoría y 3 créditos prácticos. , también habría que indicar que es la única asignatura en la que los alumnos de la Facultad de Administración y Dirección de Empresas van a ver Dirección de Operaciones.

La Dirección y Gestión de Operaciones trata acerca de la forma en que las organizaciones producen bienes y servicios. Cada objeto que utilizamos en nuestra vida cotidiana, cada tratamiento que recibimos en un hospital e incluso cada clase a la que asistimos en la universidad llegan a nosotros gracias a los Directores de Operaciones que gestionan la producción de las mismas. Esta asignatura pretende que el alumno adquiera conocicimientos específicos sobre las características fundamentales de la Dirección de Operaciones en las empresas actuales: naturaleza y contexto de la Dirección de Operaciones, la Estrategia de Operaciones de la Empresa, el Subsistema de Operaciones, el Diseño del Producto o Servicio, el Diseño del Proceso Productivo, la Distribución en Planta, la Previsión de la Demanda, la Planificación Agregada y Maestra de la Producción, la Gestión de Inventarios y la Planificación de las Necesidades de Materiales, son partes básicas de esta asignatura (Albarracín et al., 2011). Todo lo visto aquí se puede dividir en dos partes fundamentales el Diseño de un Sistema Productivo y la Gestión del mismo. Es en la primera parte, es decir, en el Diseño de un Sistema Productivo donde los profesores de la asignatura han decidido implantar el aprendizaje y la evaluación basada en proyectos esta transformación se recoge en este artículo.

\section{Objetivos}

En presente trabajo prenta los siguientes objetivos:

- Potenciar la autonomía y la responsabilidad de los alumnos así como el trabajo en equipo.

- Potenciar el pensamiento crítico y reflexivo, el sentido común (qué es el menos común de los sentidos).

- Conseguir que los alumnos aprendan haciendo.

- Conseguir que los alumnos lleven la asignatura al día.

- Mejorar las calificaciones de los alumnos de la asignatura.

\section{Desarrollo de la Innovación}

La implantación y el desarrollo de metodologías activas en el aula permite la formación en competencias de los alumnos (González y Wagemar, 2003 y Estelles-Miguel et al., 2014), lo que hace necesaria la utilización de nuevas formas de enseñanza-aprendizaje y también de nuevos sistemas de evaluación. El aprendizaje basado en proyectos pretende salvar las deficiencias del modelo de aprendizaje memorístico y mecánico y supone un buen instrumento para trabajar con alumnos que presentan competencias y habilidades distintas.

Este tipo de aprendizaje consiste en plantear un problema real a un grupo de alumnos, para cuya solución tendrán que trabajar de forma colaborativa en un proyecto que tendrán que diseñar siguiendo unas pautas iniciales marcadas por el profesor, y donde cada alumno tiene un rol individualizado con unos objetivos a conseguir (Aranda, 2009). El profesor

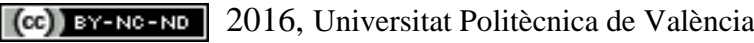

Congreso In-Red (2016) 
supervisará continuamente el estado del proyecto, pero los alumnos trabajan con una total autonomía, tomando sus propias deciones.

Para que un proyecto se lleve a cabo de forma satisfactoria será necesario establecer ciertos elementos:

- El objetivo global del proyecto.

- Los objetivos individuales de cada participante.

- Las instrucciones iniciales de cada participante.

- El tipo de evaluación final.

Este modelo de aprendizaje requiere un gran esfuerzo por parte del profesor sobre todo en la tarea previa de planificación, pero los resultados que se obtienen merecen la pena. El aprendizaje basado en proyectos pretende motivar y hacer partícipes a todos los miembros del grupo en un trabajo colaborativo, cuyos resultados son aplicables al mundo real. Lo más importante de este aprendizaje es que los alumnos puedan ver la utilidad de lo que aprenden, su aplicación a la vida real. A continuación vamos a explicar cada uno de los elementos arriba citados relacionados con el proyecto en cuestión.

\subsection{El proyecto de DPO y su objetivo}

El proyecto que se les plantea a los alumnos es la creación de una empresa de empanadillas. En primer lugar se les entrega un problema igual para todos los grupos de una empresa fictia creada por alumnos de FADE que fabrican empanadillas. Se les indica los ingredientes y cantidades de los mismos, se les indica las herramientas, máquinas, restricciones y tiempos del proceso, y se les pide calcular una serie de datos relacionados con este proceso productivo. Si se realizan bien los cálculos todos los grupos deberían obtener el mismo resultado en este primer paso.

En las siguientes clases cada uno de los grupos deberá realizar su propio proyecto de definir una empresa de empanadillas. Cada uno de los grupos está compuesto por 5 personas, cada una de las cuales tiene asignada una tarea relacionada con el proyecto, las tareas son las siguientes:

- Director.

- Responsable de Producto.

- Responsable de Proceso.

- Responsable de Distribución en Planta.

- Responsable de Localización.

El objetivo del proyecto es que cada grupo diseñe su propia empresa de fabricación de empanadillas, para lo cual deberán seguir las instrucciones que se les vayan entregando en las distintas clases.

Cada empresa puede fabricar un producto distinto (todos son empanadillas, pero los sabores, masas, formas y demás las tienen que decidir cada grupo), cada empresa tendrá un proceso de fabricación distinto dependiendo del tipo de producto que haya decidido fabricar (en función de si su variedad es muy amplia o muy estrecha, y de la cantidad de producto

(cc)) EY-NC-ND 2016, Universitat Politècnica de València 
que quieran fabricar por día, hora o turno, tendrán un tipo de proceso distinto), en base al proceso productivo que hayan definido, tendrán que seleccionar la maquinaria que necesitan para la producción del mismo y con ello realizar su distribución en planta, es decir, definir como van a colocar cada una de las máquinas, como van a estar relacionadas entre sí, cuantas personas necesitan para su proceso productivo, a cuantos turnos van a trabajar, etc.... Y finalmente con todo esto decidirán donde van a localizar su proceso productivo, y pueden buscar localizaciones reales en internet con precios, si la compran, la alquilan, etc...

\subsection{Responsabilidades y objetivos individuales}

Como hemos dicho anteriormente van a existir distintas responsabilidades en el proyecto, que serían asimilables a las existentes en una empresa real:

- Director: Será el portavoz del grupo, es el responsable de guardar el portafolio que recoge el proyecto y traerlo a clase, asegurarse de que el resto de miembros realizan las tareas asignadas, finalmente emitirá un informe del grupo, si algún día uno de los responsables no puede acudir a clase el director debe sustituirlo, tal y como ocurriría en cualquier empresa, la empresa no puede parar.

- Responsable de Producto: Deberá preparar una presentación sobre diseño de producto, y será el especialista del grupo en este tema, resolviendo todas las dudas de sus compañeros al respecto.

- Responsable de Proceso: Deberá preparar una presentación sobre diseño de proceso, y será el especialista del grupo en este tema, resolviendo todas las dudas de sus compañeros al respecto.

- Responsable de Distribución en Planta o Lay-Out: Deberá preparar una presentación sobre distribución en planta, y será el especialista del grupo en este tema, resolviendo todas las dudas de sus compañeros al respecto.

- Responsable de Localización: Deberá preparar una presentación sobre localización de instalaciones, y será el especialista del grupo en este tema, resolviendo todas las dudas de sus compañeros al respecto.

A cualquiera de los responsables de cualquier grupo se les puede solicitar que realicen una pequeña presentación, sobre una parte del tema que han preparado en profundidad y del que son expertos, durante los días que se trabaje ese punto en concreto en el aula.

\subsection{Instrucciones}

En la primera sesión se les explica a los alumnos en que va a consistir el trabajo, se les explican las tareas de los responsables y como se va a evaluar el trabajo (ver punto de evaluación).

También se explica el tema de las presentaciones de los temas. Las instrucciones para la preparación de cada presentación se darán en la semana previa a la presentación en una ficha en la que se indican contenidos mínimos de la presentacióna preparar.

Posteriormente a los grupos se les irá entregando unas fichas cada dos o tres semanas, con información adicional, relativa a la parte del trabajo que se vaya a realizar durante esas 
sesiones de clase. Se comenzará con diseño de producto, se les entrega una ficha en la que existe información de cómo hacer una empanadilla, que ingredientes pueden contener, y ese tipo de información. Ellos deben preparar ese trabajo buscando información de diferentes fuentes, realizando un brainstorming en su grupo para extraer ideas, decidiendo posteriormente de esas ideas cual de ellas podría llevarse a cabo, elaborando recetas, etc... ha habido grupos que incluso han realizado prototipos, es decir, han realizado sus propias empanadillas, horneándolas para comprobar si era factible su fabricación.

A continuación, se procedería a entregar la ficha de diseño de proceso. Indicar que para cada parte del proceso deben tener en cuenta las decisiones tomadas previamente, ya que influirán en el paso siguiente del proceso. Una vez decidan el proceso de producción deberán elegir como será la distribución en planta. Se les entregan fichas de maquinaria que pueden utilizar con todas sus características (tiempo de producción, cantidad de producción, etc), pueden buscar información adicional. Finalmente decidirán donde instalar sus instalaciones de producción y venta de producto.

\subsection{Tipo de Evaluación}

El portafolio contiene: por una parte el ejercicio inicial y por otra parte el proyecto realizado por cada uno de los grupos. Este tiene un valor de un $15 \%$ sobre la nota final de la asignatura. El portafolio lo corrige el profesor y le asignará una calificación total al trabajo que será la nota del grupo.

Por otro lado el director del grupo debe preparar un informe calificando a cada uno de sus compañeros, informando de cómo han llevado a cabo el trabajo cada uno de sus compañeros, y asignándoles una calificación de:

- A: si su desempeño ha sido sobresaliente: esto sumará 1 punto a la nota que el profesor haya dado al trabajo colectivo.

- B: si su desempeño ha sido notable: la nota será la misma que el profesor haya dado al trabajo colectivo.

- C: si su desempeño ha sido inferior al resto del grupo: esto restará 1 punto a la nota que el profesor haya dado al trabajo colectivo.

El director se puede servir de la asistencia, la participación, el trabajo realizado, etc.... Pero deberá justificar sus valoraciones. No se puede poner más de una A ni más de tres B por grupo, por cada $\mathrm{C}$ que se ponga se puede poner una A adicional.

La valoración que tenga el Director del grupo dependerá de la calidad del informe final y del orden y calidad del portafolio y del proyecto entregado.

\section{Resultados}

Los resultados obtenidos de la aplicación de esta innovación educativa son de dos tipos, por un lado la comprensión del proceso productivo. Esta parte aunque no se ha podido evaluar cuantitativamente se aprecia que los alumnos ahora comprenden mejor lo que es un producto, el proceso de diseño del mismo, los tipos de procesos productivos cuando es más conveniente utilizar uno que otro, los tipos de distribuciones en planta cuando interesa más

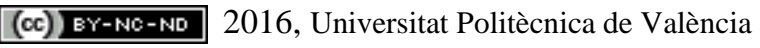


utilizar una u otra y los problemas relacionados con la localización de las instalaciones de un proceso de fabricación.

Por otro lado habría que analizar los resultados numéricos, es decir, si se ha obtenido una mejora significativa en las calificaciones de los alumnos, en cuanto a este aspecto podemos indicar dos cosas: por un lado se ha reducido ampliamente la cantidad de alumnos que suspenden esta parte de la asignatura, de hecho sólo la suspenden aquellos alumnos que abandonan la asignatura y deciden no hacer el trabajo que son casi insignificantes (de 1 a 5 por año). También habría que analizar como ha influido esto en las notas generales de la asignatura, cabría decir que las notas se han incrementado significativamente en una media de un $25 \%$ lo que nos anima a seguir con la implantación de este tipo de actividades.

\section{Conclusiones}

En la experiencia realizada, se ha demostrado que, con la elaboración de un aprendizaje basado en proyectos los alumnos aprenden haciendo y aprenden de problemas reales. Con esto aprenden a realizar trabajos colaborativos, a gestionar su tiempo y desarrollan varias habilidades clave para su futuro profesional, relacionadas con la responsabilidad individual, capacidad reflexiva, relaciones interpersonales y el trabajo en equipo.

Han mejorado las notas de los alumnos en la asignatura ascendiendo los sobresaliente en un $15 \%$ y los notables en un $17 \%$ y ha bajado el índice de aprobados.

A tenor de los resultados obtenidos podemos decir, que el cambio en el método de trabajo y de evaluación ha sido positivo. Aunque cabría indicar que la carga de trabajo realizada tanto por los profesores como por los alumnos aumenta significativamente. Para los profesores la carga que ha supuesto desarrollar el proyecto en el que van a trabajar los alumnos, el posterior seguimiento y corrección del mismo. Para los alumnos el trabajo activo a realizar en cada una de las clases, el trabajo de preparación previa a los mismos, y en el caso del director la valoración de sus compañeros.

Tras varios años de estar realizando este aprendizaje basado en proyectos nos estamos planteando la introducción de co-evaluación, es decir, que no sólo califique el director a sus compañeros sino que todos califiquen a todos, incluso que los alumnos puedan participar en la valoración de los otros trabajos presentados por el resto de grupos, creemos que este sería el siguiente paso lógico.

\section{Referencias}

ALBARRACIN, J.M, BABILONI, E. y ESTELLES-MIGUEL, S. (2011). Guía Docence de la $\begin{array}{lrrcr}\text { Asignatura Dirección } & \text { de } & \text { Producción } & y & \text { Operaciones. } \\ \text { https://docente.upv.es/GuiaDocente/inicioCursos.do?CACA=2011 } & \text { Publicado } & \text { (01/09/2011). }\end{array}$ Consultado (15/03/2016).

ARANDA, S.R. (2009). Aprendizaje basado en Proyectos. Revista Innovación Experiencias Educativas, vol. 24, pp 1-6.

BARBERA RIBERA, T., ESTELLES-MIGUEL, S. y DEMA PÉREZ, C. (2015). Student Opinicion on the Application of Active Methodologies Publicado en Sustainable Learning in Higher Education: Developing Competencies for the Global Marketplace. Editorial Springer. Suiza, pp 157-167.

(cc) EY-NC-ND 2016, Universitat Politècnica de València

Congreso In-Red (2016) 
ESTELleS-Miguel, S., BARBERA RIBERA, T y DEMA PEREZ, C. (2013). Aplicación del Portafolio Grupal en la Asignatura de Dirección de Producción y Logística: Resultados de una Pruea Piloto. Revista Educade Vol 4, pp 124-138.

ESTELleS-MIGUEL, S., RIUS SOROLLA, G y GIL, M. (2014). Designing Practical Activities for Skills Development. Publicado en Innovation and Teaching Technologies-New Directions in Research, Practice and Policy. Editorial Springer. Suiza, pp 139-147.

GARAI, GUTIERREZ y LEGARRETA (2003): Integración de las TIC en la educación superior, Pixel-Bit: Revista de medios y educación, núm. 21: 21-28.

GONZÁLEZ, J. y WAGENAAR, R. (Eds) (2003): Tuning Educational Structures in Europe. Informe Final. Fase Uno. Bilbao: Universidad de Deusto y Universidad de Groningen.

HERNÁNDEZ PINA, F. MARTÍNEZ CLARES, DA FONSECA, P y RUBIO ESPÍN, M. (2005): Aprendizaje, competencias y rendimiento en Educación Superior, Editorial La Muralla. Madrid.

LEVY-LEBOYER, C \& PRIETO, H.M. (1997): Gestión de las Competencias. Editorial Gestión.

ZABALA VIDELA, A. y ARNAU BELMONTE, L. (2007): 11 ideas clave. Cómo aprender y enseñar competencias, Colección Ideas Clave. Serie Didáctica/Diseño y desarrollo curricular. Editorial GRAÓ, de IRIF, S.L. Barcelona. 